\title{
Problemas de coordenação motora em crianças de 4 a 8 anos: levantamento baseado no relato de professores
}

\section{Problems in motor coordination in children ages 4 to 8 years old: a survey based on teachers' report}

\author{
Lívia de Castro Magalhães ${ }^{1}$, Márcia B. Rezende² ${ }^{2}$ Fernanda \\ Amparo $^{3}$, Gabriela Nunes Ferreira ${ }^{4}$, Cristiane Renger ${ }^{5}$
}

\begin{abstract}
MAGAlHÃES, L. de C.; REZENDE, M. B.; AMPARO, F.; FERREIRA, G. N.; RENGER, C. Problemas de coordenação motora em crianças de 4 a 8 anos: levantamento baseado no relato de professores. Rev. Ter. Ocup. Univ. São Paulo, v. 20, n. 1, p. 20-28, jan./abr. 2009.

RESUMO: O termo transtorno do desenvolvimento da coordenação (TDC) ainda é pouco usado no Brasil e a maioria das crianças com esse tipo de problema não é diagnosticada. As dificuldades de coordenação motora geralmente são evidenciadas na escola e, embora queixas de crianças com problemas de escrita ou lentidão motora sejam comuns, essas dificuldades não são identificadas como uma questão que merece atenção especial. O objetivo deste estudo foi examinar a freqüência e as atividades nas quais professores observam dificuldades de coordenação motora em crianças de 4 a 8 anos de idade. Um questionário simples, com questões fechadas, foi enviado a escolas da região metropolitana de Belo Horizonte. Dados de 288 questionários, sendo 148 de professores da rede Municipal e 140 de escolas particulares, foram analisados descritivamente. A maioria dos professores, $88 \%$ nas escolas Municipais e $79 \%$ nas particulares, indicaram que geralmente observam problemas de coordenação em um a dois alunos em cada classe, o que resultou na estimativa de $18,6 \%$ de crianças com algum tipo de dificuldade motora. Estes dados superam os $6 \%$ reportados na literatura internacional, sendo importante investir em estudos epidemiológicos e na identificação correta do TDC em crianças brasileiras.
\end{abstract}

PALAVRAS-CHAVE: Desenvolvimento infantil. Crianças. Transtorno das modalidades motoras. Questionário/utilização. Docentes. Saúde escolar.

\footnotetext{
1. Profa. Titular, Departamento de Terapia Ocupacional, Universidade Federal de Minas Gerais.

2. Profa. Associada, Departamento de Terapia Ocupacional, Universidade Federal de Minas Gerais.

3. Terapeuta Ocupacional, Coordenadoria de Doenças Complexas, Secretaria de Estado de Saúde de Minas Gerais.

4. Terapeuta Ocupacional, Centro Geral de Pediatria, Fundação Hospitalar de Minas Gerais.

5. Terapeuta Ocupacional, Habilitar Núcleo de Desenvolvimento e Saúde, Belo Horizonte, MG.

Endereço para correspondência: Profa. Lívia de Castro Magalhães: Escola de Educação Física, Fisioterapia e Terapia Ocupacional/ DTO. Avenida Antônio Carlos 6627. Bairro Pampulha. Belo Horizonte-MG, CEP 31270-901. Telefone: (31) 3409-4790, e-mail: liviam@ gcsnet.com.br
} 
s dificuldades de coordenação motora, comuns
em crianças em idade escolar, têm ocupado
lugar de destaque na literatura internacional, sendo consideradas, por alguns autores, como um dos grandes problemas de saúde para a criança (CARNEY at al., 2005; GREEN et al., 2005; HENDERSON; HENDERSON, 2002; POLATAJKO; CANTIN, 2006). No Brasil, apesar de na prática clínica nos deparamos com queixas freqüentes das professoras, este assunto ainda é pouco pesquisado. Cientes das complexidades inerentes à questão da educação em nosso país, não se pretende, como criticado por alguns autores (MOYSÉS; COLLARES, 1997), descrever mais um diagnóstico clínico e elegê-lo como causa do fracasso escolar. É evidente que não se pode centrar a questão do fracasso escolar apenas no aprendiz, pois, em um país com tantas desigualdades como o Brasil, um conjunto de condições socioculturais e econômicas dificulta ou mesmo impossibilita a inserção de grande contingente de alunos nos processos de aprendizagem escolar (GRIFFO, 2000). Essas condições com certeza merecem atenção, no entanto, independente da perspectiva adotada, não se pode ignorar que muitas crianças apresentam déficits motores relevantes, que dificultam sua participação nas várias atividades escolares (MISSIUNA et al., 2004; STEPHENSON; CHESSON, 2008).

Alterações na coordenação motora estão associadas a vários distúrbios do sistema nervoso central, mas, na criança, existe uma condição em que, na ausência de sinais específicos de lesão cerebral, observa-se dificuldade no desempenho de tarefas funcionais. São crianças inteligentes, mas que apresentam dificuldades para realizar tarefas simples, exigidas no cotidiano escolar, como apontar lápis, escrever sem sair da linha, recortar, agarrar uma bola ou correr no ritmo dos colegas. Tais dificuldades, além de prejudicar o desempenho na escola, influenciam negativamente a auto-estima e o senso de competência da criança (CARNEY et al., 2007; DEWEY et al., 2002; SEGAL et al., 2002).

Este tipo de transtorno motor já recebeu diversos nomes, tais como disfunção cerebral mínima (DCM), síndrome psicomotora, paralisia cerebral mínima, dispraxia do desenvolvimento, somatodispraxia, síndrome da criança desajeitada ("clumsy child") e DAMP (MAGALHÃES et al., 2006). Atualmente, no entanto, o termo mais usado é "Transtorno do Desenvolvimento da Coordenação" - TDC (ou Developmental Coordination Disorder - DCD). Este termo apareceu pela primeira vez no Manual Estatístico de Diagnóstico de Distúrbios Mentais (DSM III-R), da Associação Americana de Psiquiatria (APA) em 1987 e foi mantido no DSM-IV sob o código 315.4. O DSM-IV especifica que o diagnóstico de TDC se aplica aos casos em que, na ausência de distúrbios físicos ou neurológicos conhecidos, observa-se desempenho abaixo do esperado para a idade cronológica e nível cognitivo da criança nas atividades diárias que requerem coordenação motora.

Em sua versão atual, o DSM-IV-TR (APA 2002) apresenta três critérios para o diagnóstico de TDC: (a) o transtorno motor deve interferir de maneira significativa no desempenho de atividades cotidianas ou escolares, (b) a dificuldade de coordenação não pode ser devida a condições médicas ou a transtorno invasivo do desenvolvimento, e (c) caso haja retardo mental associado, as dificuldades motoras devem ser maiores que as esperadas em função do retardo. O TDC também é reconhecido como entidade nosológica na Classificação Internacional de Doenças (CID-10) da Organização Mundial de Saúde (OMS, 2003). O CID-10 adota o temo "Transtorno Específico do Desenvolvimento da Função Motora” (F 82), mas não especifica como critério diagnóstico o prejuízo acadêmico ou no desempenho de tarefas diárias, porém preconiza o uso de testes padronizados de coordenação motora fina e grossa para se estabelecer o diagnóstico.

A causa do TDC ainda não é bem esclarecida, no entanto, acredita-se que seja multifatorial. Existem estudos sobre a correlação entre anormalidades observadas em ressonância magnética ou na tomografia computadorizada e a coordenação motora, mas os dados não são conclusivos (BARNETT et al., 2002); outros autores discutem a relação entre prejuízos cerebrais, complicações perinatais, prematuridade, baixo peso ao nascimento e fatores familiares com o TDC (FAWKE, 2007; JONGMANS et al., 1998; MAGALHÃES et al., no prelo). Sabe-se, também, que os problemas motores não constituem uma condição isolada, sendo freqüente a associação com as dificuldades de aprendizagem e o transtorno do déficit de atenção e hiperatividade (KAPLAN et al., 2006; DEWEY et al., 2002; GREEN; BAIRD, 2005).

Os sinais característicos do TDC são desajeitamento e inconsistência no desempenho de tarefas motoras, coordenação motora pobre, problemas de ritmo e na transferência de aprendizagem, declínio do desempenho com a repetição, aumento na tensão corporal e excesso de atividade muscular durante a execução de tarefas motoras (CERMAK et al., 2002). Observa-se, também, que estas crianças podem apresentar atraso significativo nos marcos do desenvolvimento motor (ex.: engatinhar, ficar de pé, andar) e tendem a mostrar pouco interesse por esportes e brincadeiras que exijam coordenação motora grossa ou fina mais apurada (CAIRNEY et al., 2007). Além da dificuldade nas atividades do auto-cuidado e nas tarefas 
escolares, muitas crianças com TDC também têm problemas na participação social, uma vez que habilidades motoras são requisito para várias brincadeiras infantis e crianças com lentidão motora, muitas vezes, são excluídas pelos colegas (MANDICH et al., 2003; POULSEN et al., 2008; STEPHENSON; CHESSON, 2008).

Observa-se, portanto, que o TDC não é simplesmente um transtorno motor, pois há implicações psicossociais importantes. Estudos longitudinais indicam que os problemas de coordenação motora não são quadros transitórios, típicos apenas da infância, pois podem ter impacto na idade adulta (CANTELL; SMITH, 2003; COUSINS; SMITH, 2003), havendo relato de maior frequência de casos de depressão, desajuste social e problemas emocionais entre indivíduos com TDC (HELLGREEN et al., 1994; RASMUSSEN; GILBERG, 2000; PIEK et al., 2006).

A literatura internacional aponta que entre 5 a $10 \%$ das crianças em idade escolar apresentam TDC (APA, 2002; CAIRNEY et al., 2007). No Brasil, não há dados epidemiológicos conclusivos, mas Souza e colaboradores (2006) encontraram sinais de TDC em 4,4 a $11,8 \%$ de crianças da zona urbana e rural, respectivamente, da região norte do país. Considerando que o TDC é um problema freqüente na infância, mas ainda pouco pesquisado em nosso país, o objetivo do presente estudo foi documentar a freqüência com que professores de educação infantil e séries iniciais do ensino fundamental observam problemas de coordenação motora nas crianças na sala de aula. A meta foi de registrar sinais de problemas motores, em atividades escolares típicas, sem o objetivo de fazer diagnóstico específico das crianças. Além da freqüência de crianças com sinais de problemas na coordenação motora e das atividades escolares na quais se observa mais dificuldade, foi feito levantamento das ações dos professores quando deparam com o problema. Uma vez que professores de escolas públicas e particulares têm acesso à populações infantis diferentes, nesse estudo foram examinados dados coletados nesses dois contextos.

\section{METODOLOGIA}

\section{Participantes}

Os participantes deste estudo foram professoras de crianças com idades entre 4 e 8 anos, de escolas das redes Municipal e particular da Região Metropolitana de Belo Horizonte. Essa faixa etária foi selecionada por ser um período crítico para deteç̧ão de problemas de coordenação motora. É na fase pré-escolar e inicio do ensino fundamental que aparecem as primeiras queixas de dificuldade escolar relacionada a problemas motores, sendo importante documentar em que tipo de atividade as professoras começam a observar dificuldades. Foram recrutadas professoras com no mínimo dois anos de experiência didática, envolvidas com o ensino de turmas regulares, com no mínimo oito alunos em sala. Foram excluídos os questionários referentes a turmas de ensino especial, assim como os dados de crianças com sinais de desenvolvimento atípico, síndromes genéticas ou qualquer diagnóstico neurológico.

\section{Instrumentação}

O instrumento utilizado para a coleta de dados deste estudo consistiu em questionário simples, com perguntas fechadas, no qual o professor inicialmente assinalava os anos de experiência, o nível escolar da turma sob sua responsabilidade, número de alunos e número de crianças com qualquer tipo de síndrome ou diagnóstico neurológico. Registrado o número de crianças com necessidades especiais, o professor era instruído a focar a atenção no restante da turma, para responder ao questionário tendo como base o comportamento das crianças sem qualquer diagnóstico clínico. O questionário incluiu questões referentes ao desempenho das crianças em atividades de coordenação motora fina e grossa, número de crianças com sinais de dificuldade motora e conduta da professora face aos problemas observados. No início do questionário havia um parágrafo explicativo sobre os objetivos do projeto e, ao final, foi deixado pequeno espaço para comentários.

\section{Procedimentos}

Estudo descritivo transversal, operacionalizado por meio de aprovação da Secretaria Municipal de Educação da cidade de Belo Horizonte para coleta de dados na Rede Municipal de ensino. Escolas da rede particular foram contatadas individualmente, sendo coletada amostra de conveniência em escolas receptivas ao projeto e cuja localização facilitasse a entrega e coleta dos questionários. Em ambas as redes de ensino, procurou-se abranger as nove regionais do município de Belo Horizonte. Uma vez aprovado o projeto nas escolas, os questionários foram encaminhados ao setor de orientação pedagógica e repassados às professoras. Os questionários foram preenchidos pelas professoras que, cientes dos objetivos do estudo, se dispuseram a participar da pesquisa. Os questionários referentes às diferentes turmas foram 
retornados ao setor de orientação pedagógica de cada escola e recolhidos pela equipe de pesquisa, cerca de uma semana após a entrega. Parte dos dados das Escolas Municipais foi coletada em curso de atualização para professores da rede pública, oferecido pela Secretaria Municipal de Educação. As professoras não receberem nenhum tipo de treinamento para preencher o questionário, uma vez que as questões eram simples e o objetivo não foi de detectar problemas específicos de coordenação motora, mas sim de levantar possíveis dificuldades motoras observadas espontaneamente pelas professoras.

Em termos de organização, na rede Municipal, as turmas são divididas de acordo com a idade das crianças, havendo uma estimativa de 1400 professores para crianças de 4 a 8 anos de idade. Na rede particular, as turmas são distribuídas por nível de desempenho, não havendo estimativa precisa do número total de professores. O projeto foi aprovado pelo Comitê de ética em Pesquisa COEP/ UFMG.

\section{Análise dos dados}

Os dados coletados no estudo foram analisados pelo pacote estatístico SPSS Versão 11.0. Análise descritiva foi usada para caracterizar a amostra e teste-t foi usado para examinar possíveis diferenças entre professores de escolas públicas e particulares, quanto às atividades nas quais são observados problemas de coordenação motora.

\section{RESULTADOS}

Um total de 288 professores de crianças de 4 a 8 anos de idade, sendo 148 da rede pública Municipal e 140 da rede particular participou do estudo. A taxa de retorno dos questionários variou de $90 \%$ nas escolas particulares a $40 \%$ nas escolas municipais e $10 \%$ dos questionários foram descartados devido a dados incompletos. Algumas escolas abordadas recusaram-se a participar do estudo. A amostra representa cerca de $10 \%$ do número de professores da Rede Municipal. Foram coletados dados das nove regiões metropolitanas, havendo maior volume de questionários preenchidos $(63,1 \%)$ de escolas municipais de regiões menos afluentes (i.e., Venda Nova, Pampulha e Barreiro) e maior volume de dados $(68,6 \%)$ de escolas particulares de regiões típicas de classe média (i.e., centro-sul, noroeste e nordeste), o que reflete a distribuição desses dois tipos de escola na região metropolitana de Belo Horizonte.

A Tabela. 1 apresenta a distribuição dos professores de acordo com o nível de idade das turmas. O número de alunos por turma nos dois sistemas escolares foi semelhante, com número significativamente maior de alunos nas escolas municipais apenas nas turmas de 5 e 6 anos, mas o maior desvio padrão (DP) nas escolas particulares sinaliza aumento no número médio de estudantes por classe nas turmas mais avançadas. Deve-se observar que, como a implantação de programas para crianças de 4 a 5 anos na Rede Municipal começou cerca de dois anos antes da coleta de dados, houve menor número de questionários respondidos por professores de Escolas Municipais nessa faixa etária.

TABELA 1. Número de professores e distribuição de alunos por turma

\begin{tabular}{lcccc}
\hline & \multicolumn{2}{c}{ Escolas Municipais } & \multicolumn{2}{c}{ Escolas Particulares } \\
\cline { 2 - 5 } Turmas & $\begin{array}{c}\text { Número de } \\
\text { professores }\end{array}$ & $\begin{array}{c}\text { Número médio de } \\
\text { alunos/turma (DP) }\end{array}$ & $\begin{array}{c}\text { Número de profes- } \\
\text { sores }\end{array}$ & $\begin{array}{c}\text { Número médio de } \\
\text { Alunos/turma (DP) }\end{array}$ \\
\hline 4 anos & 11 & $18,9( \pm 3,2)$ & 28 & $18,2( \pm 5,1)$ \\
5 anos & 12 & $22,6( \pm 1,9)^{*}$ & 31 & $18,4( \pm 5,0)$ \\
6 anos & 42 & $24,1( \pm 2,2)^{*}$ & 30 & $21,2( \pm 5,3)$ \\
7 anos & 42 & $25,6( \pm 2,3)$ & 34 & $26,9( \pm 5,5)$ \\
8 anos & 38 & $25,3( \pm 3,5)$ & 14 & $24,4( \pm 6,5)$ \\
& 148 & 23,2 & 140 & 21,8 \\
\hline
\end{tabular}

Nota: DP $=$ Desvio padrão, * t-test, diferença significativa $\mathrm{p}>0,05$.

Os dados indicaram que $63 \%$ dos professores das escolas públicas e particulares observam problemas de coordenação motora nas classes de crianças de 4 a 8 anos de idade, sendo que $88 \%$ dos professores de escolas públicas 
e $79 \%$ de professores de escolas particulares relatam sempre observar uma ou duas crianças com problemas de coordenação motora em suas salas de aula. Quando perguntado, 65,3\% dos professores da Rede Municipal e $61,5 \%$ dos professores da Rede Particular indicam acreditar que crianças com problema de coordenação motora têm maior dificuldade de alfabetização. Nos Gráficos 1 e 2 são apresentadas as freqüências com que professores de escolas públicas e particulares observam dificuldade no desempenho de atividades de coordenação motora grossa e fina.

GRÁFICO 1. Porcentagem de professores que observam dificuldade nas atividades de coordenação motora grossa

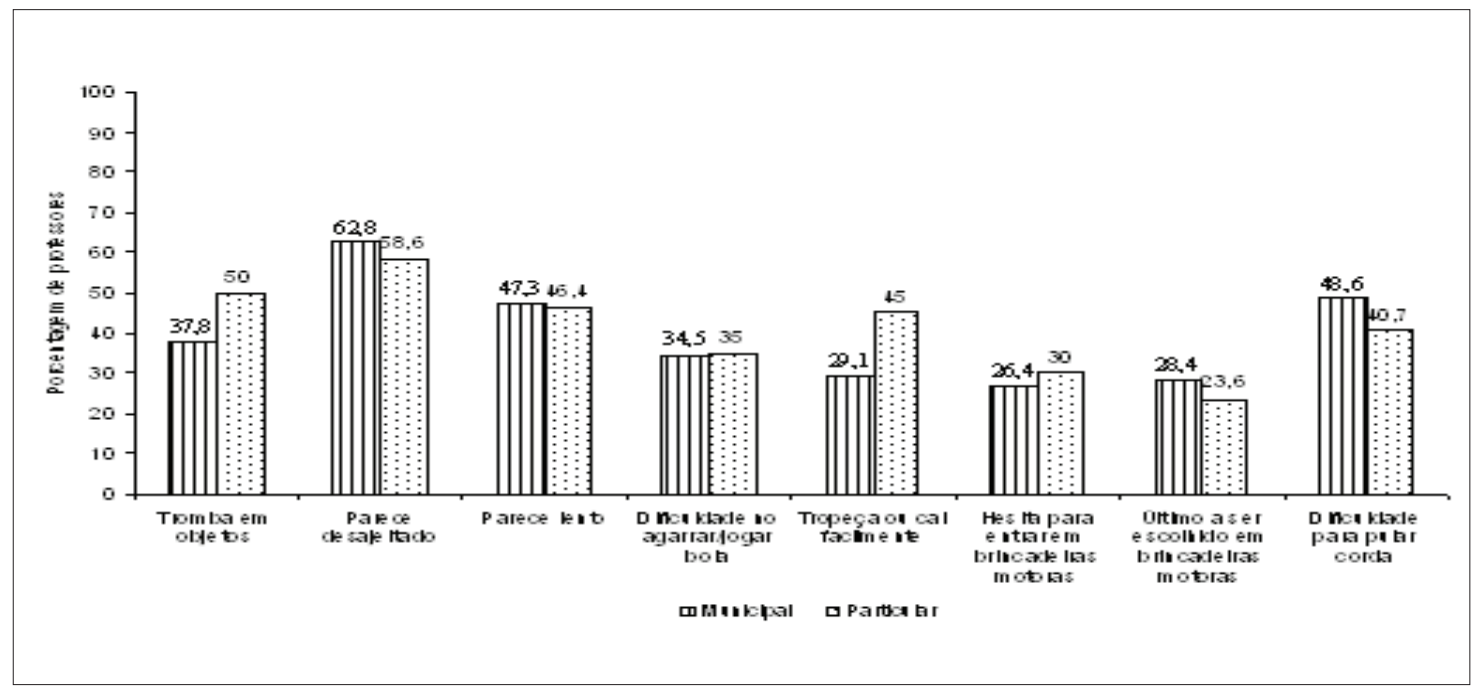

GRÁFICO 2. Porcentagem de professores que observam dificuldade nas atividades de coordenação fina

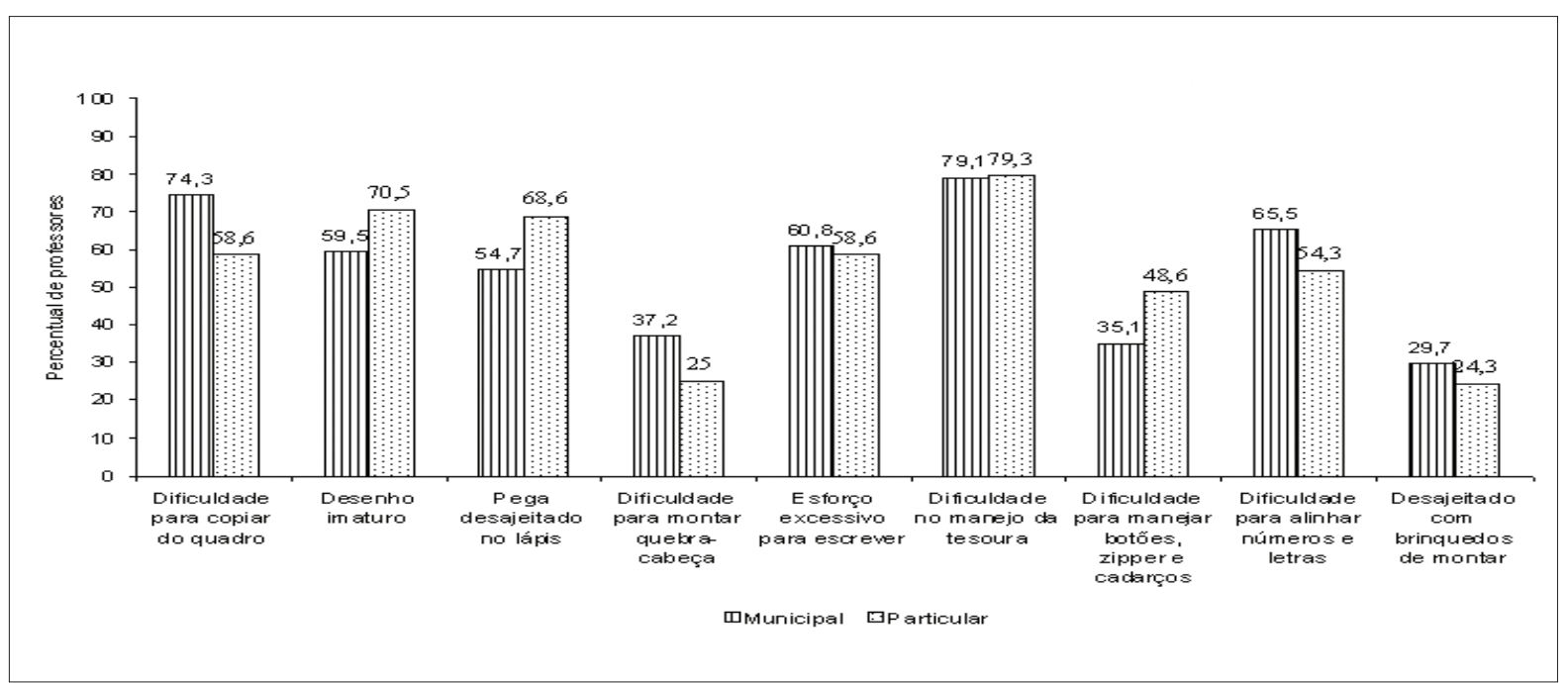


Fazendo somatório do número de crianças registradas pelas professoras como apresentando problemas de coordenação fina, grossa ou ambos, observou-se que nas escolas municipais a freqüência foi de $20,5 \%$ e nas particulares foi de $16,8 \%$, o que resultou em média global de $18,6 \%$ de crianças com algum tipo de dificuldade de coordenação motora nas escolas examinadas. Como o somatório do número de alunos por turma, reportado pelas professoras, indica que os dados da pesquisa abrangem um total de 6.496 crianças, estima-se que $1.208(18,6 \%)$ delas necessitam de algum suporte ou avaliação diagnóstica, devido a problemas de coordenação motora. Além das crianças com sinais de problemas de coordenação motora, as professoras reportam a presença de $99(1,5 \%)$ de crianças com necessidades especiais.

Com relação à atitude da professora para com alunos com dificuldade de coordenação motora, a maioria procura resolver questão dentro da escola, seja procurando o setor de orientação pedagógica, $66 \%$ das professoras nas escolas públicas e $92 \%$ nas particulares, ou dando mais atenção à criança, 65,3 e 56,2\% nas escolas públicas e particulares, respectivamente. A maioria das professoras reportou conversar com os pais (58,5 nas públicas e 73,5\% nas particulares) e poucas professoras, 16,3 das públicas e $27,7 \%$ das particulares, reportaram que encaminham a criança para tratamento. Algumas professoras de escolas municipais comentaram, por escrito, que não encaminham crianças com problemas de coordenação motora para diagnóstico, avaliação ou terapia porque esses serviços não estão disponíveis no sistema público e eles não saberiam para qual profissional seria mais adequado encaminhar.

\section{DISCUSSÃO}

Os resultados indicam que as professoras observam problemas de coordenação motora e que, na amostragem examinada, número significativo $(18,6 \%)$ das crianças matriculadas no ensino infantil e séries iniciais do ensino fundamental apresentava sinais de algum tipo de dificuldade motora. Embora não se possa afirmar que essas crianças tenham transtorno da coordenação motora, pois não foi feita avaliação específica, essa estimativa é muito superior à reportada em estudo recente, no Canadá, que indica prevalência de 7,5\% de TDC em crianças de 9 a 14 anos (CAIRNEY et al., 2007).

Ficou evidente, também, que as professoras tendem a observar mais problemas na área de coordenação fina (Gráficos 1 e 2), o que parece relacionado ao fato das atividades de sala de aula requerem mais esse tipo de habilidade. Embora, com base apenas no relato das professoras, não seja possível saber se as crianças identificadas apresentam ou não TDC, os dados são preocupantes. Em primeiro lugar porque sinalizam para a possibilidade de prevalência acima dos 7\% reportada internacionalmente (APA, 2002; CAIRNEY et al., 2007). Esse é um dado importante, pois ainda não existem estudos epidemiológicos sobre o TDC em países em desenvolvimento, sendo que, problemas nutricionais, mais comuns entre crianças de escolas públicas como as reportadas no presente estudo, estão entre os fatores de risco para atraso no desenvolvimento motor. A pequena diferença, de cerca de quatro pontos percentuais, no índice de professores de escolas municipais e particulares que reportam problemas de coordenação motora nos alunos, sugere, como esperado, maior freqüência desse tipo de problema entre crianças em desvantagem social.

Um ponto que merece destaque é que, devido ao regime de meio período nas escolas do Brasil, as professoras geralmente têm oportunidade para observar a criança apenas na sala de aula, o que pode ter limitado sua habilidade para julgar o desempenho em atividades de coordenação grossa (Gráfico 1). Segundo Geuze (2002) o diagnóstico de TDC inclui um grupo heterogêneo de crianças, sendo importante examinar os vários aspectos motores. O TDC está intimamente relacionado aos problemas de escrita, que têm relação direta com o rendimento escolar, no entanto, embora geralmente menos valorizado pelas professoras, $\mathrm{o}$ pobre desempenho em esportes e atividades físicas típicas para a idade, pode levar à exclusão social e baixa autoestima, com impacto no desempenho escolar (CARNEY et al., 2005). No presente estudo, os dados indicam que seria interessante promover maior familiarização das professoras sobre as características do TDC, estimulando assim, a observação da criança nos diferentes contextos, incluindo o recreio e as aulas de educação física.

Independente do índice exato de prevalência, os dados chamam a atenção para o fato de que, tanto professoras de escolas públicas quanto particulares, com diferentes formações e perspectivas teóricas sobre educação, reportam que lidam rotineiramente com crianças com algum tipo de dificuldade de coordenação motora, sendo importante usar recursos que permitam melhor identificação dessas crianças. Testes de triagem para TDC, no formato de questionários de fácil aplicação, são muito usados no exterior (HAY et al., 2004; WILSON et al., 2000) e constituem recurso útil para identificar crianças que necessitam de maior atenção ou suporte extra-classe.

As atitudes que os professores tomam quando se deparam com o problema de coordenação motora na sala de aula parecem mais centradas em recursos didáticos e estratégias pedagógicas, do que em abordagens motoras 
específicas, que poderiam ser mais benéficas para crianças que têm problemas de coordenação motora. Seria interessante comparar a eficácia de estratégias pedagógicas e motoras na melhoria do desempenho escolar de crianças com TDC, mas isso ainda não foi examinado na literatura. Um aspecto interessante, mencionado por algumas professoras, é que elas não têm clareza sobre o tipo de programa de intervenção ou profissional que poderia ajudar essas crianças. Considerando o ensino público, as alternativas são poucas, pois na maioria dos casos as escolas não contam com suporte de equipes de saúde, que poderiam ajudar pelo menos no diagnóstico da criança. Embora no ensino particular mais professoras recorram ao encaminhamento para profissionais especializados (27\%), esse número ainda é pequeno, indicando o desconhecimento ou a pouca confiança em recursos fora da escola.

Considerando o grande número de crianças que apresentam sinais de dificuldade nas atividades que exigem coordenação motora, 1.208 só no presente estudo, não se pode ignorar o problema. Acreditamos que não é viável pensar, como sugerido por algumas professoras, em modelos de intervenção centrados no encaminhamento a profissional especializado, pois, além de alto custo e do justo antagonismo à medicalização da educação (MOYSÉS; COLLARES, 1997), dificuldades individuais só podem ser compreendidas dentro do próprio contexto escolar. É importante, assim, criar estratégias de atenção à criança que possam ser incorporadas à rotina escolar, $\mathrm{o}$ que inclui programas de consultoria e orientação para pais e professores, para identificar o problema e dar suporte à criança, tanto na escola como em casa (MISSIUNA et al., 2004; SUGDEN; CHAMBERS, 2003).

É interessante ressaltar que a taxa de retorno dos questionários nas escolas Municipais (40\%) sugere que os problemas de coordenação motora não constituem tema de grande interesse entre os professores destas escolas, apesar do número significativo de crianças $(20,5 \%)$ que apresentam sinais de dificuldades motoras. Nos parece importante fornecer mais informações sobre o TDC para os professores (MISSIUNA, 2007), para estimular o interesse e melhorar a compreensão sobre recursos e estratégias práticas, que podem ser usados em sala de aula, para minimizar as dificuldades enfrentadas por essas crianças.

Outro dado relevante, embora não diretamente relacionado à questão do TDC, é que a inclusão de crianças com incapacidades ainda parece ser um ideal a ser alcançado, pois na amostragem em estudo, o número de crianças com necessidades especiais que freqüentavam as escolas pesquisadas foi pequeno. $\mathrm{O}$ diagnóstico da maioria dessas crianças era desconhecido, o que denota pouco entrosamento entre os serviços de saúde e educação.

\section{CONCLUSÃo}

Sinais de problemas de coordenação motora em crianças de 4 a 8 anos de idade parecem evidentes na sala de aula, sendo observados por professoras das redes Municipal e particular. Como os dados sugerem que cerca de $18 \%$ de crianças nas escolas examinadas apresentam algum tipo de dificuldade motora, caracteriza-se um problema de saúde pública, que merece estudos epidemiológicos, sobretudo porque o TDC pode ter conseqüências importantes na adolescência e idade adulta, que transcendem o ambiente escolar. Embora apoio pedagógico na sala de aula possa beneficiar muitas crianças, é importante também considerar o papel da orientação específica a pais e professores, que podem aprender estratégias e fazer adaptações para minimizar as demandas motoras nas tarefas escolares.

Um grande empecilho para a oferta de atenção mais individualizada ao transtorno da coordenação é a identificação das crianças que realmente apresentam este problema. É importante investir em recursos para identificação do TDC, o que vem sendo feito em outros estudos (PRADO et al., 2009), assim como na educação de professores e pais, para que eles entendam as dificuldades da criança e possam dar o suporte apropriado para facilitar a participação social e o sucesso em todas as atividades escolares. Esse é um excelente campo de atuação para o terapeuta ocupacional, que vem sendo explorado em outros países (MISSIUNA et al., 2008). $\mathrm{O}$ terapeuta ocupacional, além de ajudar na identificação e esclarecimento sobre o que é o TDC, deve contribuir com estudos epidemiológicos, que ajudem a entender melhor o problema e suas conseqüências a longo prazo. $\mathrm{Na}$ área de intervenção, considerando o volume de crianças com dificuldades motoras, enfatizamos a necessidade de se investir em modelos de consultoria, centrados no estabelecimento de relações de parceria com as professoras, visando colaborar no planejamento de estratégias efetivas de sala de aula para dar suporte ao bom desempenho escolar.

AgRAdecimento: Agradecemos o apoio da Secretaria Municipal de Educação de Belo Horizonte, em especial à Dra. Socorro Nunes, e das diretoras das escolas Municipais e particulares que participaram do estudo. Agradecemos às professoras que colaboraram preenchendo os questionários, pois sem sua ajuda esse estudo não teria sido possível. Agradecemos também à Fundação de Amparo à Pesquisa do Estado de Minas Gerais - FAPEMIG (CDS 80511/04), pelo suporte financeiro ao projeto. 
MAGAlHÃES, L. de C. et al. Problemas de coordenação motora. Rev. Ter. Ocup. Univ. São Paulo, v. 20, n. 1, p. 20 -28, jan./abr. 2009.

MAGALHÃES, L. de C.; REZENDE, M. B.; AMPARO, F.; FERREIRA, G. N.; RENGER, C. Problems in motor coordination in children ages 4 to 8 years old: a survey based on teachers' report.

Rev. Ter. Ocup. Univ. São Paulo, v. 20, n. 1, p. 20-28, jan./abr. 2009.

\begin{abstract}
The term developmental coordination Disorder (DCD) is still new in Brazil and most of the children with this type of problems are not diagnosed. Motor coordination difficulties usually are observed at school, and although complaints about children with handwriting problems or motor awkwardness are quite common, these difficulties are not identified as a problem that deserves special attention. The objective of this study was to examine the frequency and in which activities do teachers observe motor coordination problems among children ages 4 to 8 years old. A simple questionnaire, with direct questions, was sent to schools of the metropolitan region of Belo Horizonte. Data from 288 questionnaires, 148 from teachers of public schools and 140 from private schools, were analyzed descriptively. The majority of the teachers, $88 \%$ from the public schools and $79 \%$ from the private ones, reported that they usually observe one or two students with motor coordination problems in each class they teach, which resulted in an overall estimate of $18,6 \%$ children with some sort of motor difficulty. This result exceeds the $6 \%$ reported in the international literature; therefore, it is important to invest in epidemiological studies and in the correct identification of DCD among the Brazilian children.
\end{abstract}

KEY WORDS: Child development. Child. Motor skills disorders. Questionnaire/utilizaton. Faculty. School health.

\title{
REFERÊNCIAS
}

AMERICAN PSYCHIATRIC ASSOCIATION. Manual diagnóstico e estatístico de transtornos mentais: DSM-IV-TR tm - texto revisado. 4. ed. rev. Porto Alegre: Artmed, 2002.

BARNETT, A.; MERCURI, E.; RUTHERFORD, M.; HAATAJA, L.; FRISONE, M. F; HENDERSON, S. et al. Neurological and perceptual-motor outcome at 5-6 years of age in children with neonatal encephalopathy: relationship with neonatal brain MRI. Neuropediatrics, v. 33, n. 5, p. 242-248, 2002.

CAIRNEY, J.; HAY, J.; MANDIGO, J. J.; WADE, T.; FAUGHT, B. E.; FLOURIS, A. Developmental coordination disorder and reported enjoyment of physical education in children. Eur. Phys. Educ. Rev., v. 13, p. 81-98, 2007.

CAIRNEY, J.; HAY, J. A.; FAUGHT, B. E.; HAWES, R. Developmental coordination disorder and overweight and obesity in children aged 9-14y. Int. J. Obes., v. 29, n. 4, p 369-372, 2005.

CANTELL, M. H.; SMYTH, M. M.; AHONEN, T. P. Two distinct pathways for developmental coordination disorder: Persistence and resolution. Hum. Mov. Sci., v. 22, n. 4-5, p. 413-431, 2003.

CERMAK, S.A.; GUBBAY, S.S., LARKIN, D. What is developmental coordination disorder? In: CERMAK, S. A.; LARKIN, D. (Orgs.). Developmental coordination disorder. New York: Albany, 2002. p. 2-22.

COUSINS, M.; SMYTH, M.M. Developmental coordination impairments in adulthood. Hum. Mov. Sci., v. 22, n. 4-5, p. $433-$ 459, 2003.

DEWEY, D.; KAPLAN, B. J; CRAWFORD, S. G; WILSON, B. N. Developmental coordination disorder: associated problems in attention, learning, and psychosocial adjustment. Hum. Mov. Sci., v. 21, n. 5-6. p. 905-918, 2002.

DUNFORD, C.; MISSIUNA, C.; STREET, E.; SIBERT, J. Children's perceptions of the impact of developmental coordination disorder on activities of daily living. Br. J. Occup. Ther., v. 68, n. 5, p. 207-214, 2005.

GEUZE, R. H.; JONGMANS, M.; SCHOEMAKER, M. M.; BOUWIEN, C. M.; SMITS-ENGELSMAN, B. C. Clinical and research diagnostic criteria for developmental coordination disorder: a review and discussion. Hum. Mov. Sci., v. 20, p. 7- 47, 2001.

GREEN, D.; BAIRD, G. DCD and overlapping conditions. In: SUGDEN, D.; CHAMBERS, M., organizadores. Children with developmental coordination disorder. Philadelphia: Whurr Publishers, 2005. p. 93-118.

GRIFFO, C. Dificuldades de aprendizagem na alfabetização: perspectivas do aprendiz. In: GOMESS, M. F. C.; SENA, M. G. C.(Orgs.). Dificuldades de aprendizagem na alfabetização. Belo Horizonte: Autêntica Editora, 2000. p.39-54.

GREEN, D.; BISHOP, T.; WILSON, B. N.; CRAWFORD, S.; 
HOOPER, R.; KAPLAN, B. J. et al. Is question-based screening part of the solution to waiting lists for children with developmental coordination disorder? Br. J. Occup. Ther., v. 68, n. 1, p. 1-9, 2005.

HAY, J. A.; HAWES, R., FAUGHT, E. Evaluation of a screening instrument for developmental coordination disorder. J. Adolesc. Health, v. 34, n. 4, p. 308-313, 2004.

HellgRen, L.; GILlBERG, IC.; BAGENhOlm, A.; GILLBERG, C. Children with deficits in attention, motor control and perception (DAMP) almost grown up: psychiatric and personality disorders at age 16 years. J. Child. Psychol. Psychiatry, v. 3, p. 1255-1271, 1994.

HENDERSON, S. E.; HENDERSON, L. Toward an understanding of developmental coordination disorder. Adapt. Phys. Activ. Q., v.19, n. 1, p. 12-31, 2002.

JONGMANS, M. J.; MERCURI, E.; DUBOWITZ, L.; Henderson, S. Perceptual-motor difficulties and their concomitants in sixyear-old children born prematurely. Hum. Mov. Sci., v. 17, p. 629-653, 1998.

KAPLAN, B. J.; CRAWFORD, S. G.; CANTELL, M.; KOOISTRA, L.; DEWEY, D. Comorbity, co-occurrence, continuum: what's in a name? Child Care Health Develop., v. 32, n. 6, p. 723-731, 2006.

MAGALHÃES, L. C.; REZENDE, F. C.; MAGALHÃES, C.M.; ALBUQUERQUE, P. D. R. Coordenação motora em crianças brasileiras a termo e pré-termo aos 7 anos de idade. Rev. Bras. Saude Mater. Infant., no prelo.

MAGALHÃES, L. C.; MISSIUNA, C.; WONG, S. Terminology used in research reports of developmental coordination disorder. Dev. Med. Child Neurol., v. 48, n. 11, p. 937-41, 2006.

MANDICH, A.D.; POLATAJKO, H.J.; RODGER, S. Rites of passage: Understanding participation of children with developmental coordination disorder. Hum. Mov. Sci., v. 22, n. 4-5, p. 583-595, 2003.

MISSIUNA, C.; POLLOCK, N.; EGAN, M.; DELAAT, D.; GAINES, R.; SOUCIE, H. Enabling occupation through facilitating the diagnosis of developmental coordination disorder. Can J. Occup. Ther., v. 75, n. 1, p. 26-34, 2008.

MISSIUNA, C.; RIVARD, L.; POLLOCK, N. They're bright but can't write: Developmental coordination disorder in school aged children. Teaching Exceptional Children Plus, v. 1, n. 3, p. $1-11,2004$.

MISSIUNA C. Children with developmental coordination disorder: at home and in the classroom. Disponível em: <http:// www.fhs.mcmaster.ca/canchild/>. Acesso em: 23 out. 2007.
MOYSÉS, M. A.; COLLARES, C. A. Inteligência abstraída, crianças silenciadas: as avaliações de inteligência. Rev. Psicol. $U S P$, v. 8, n. 1, p. 63-89,1997.

ORGANIZAÇÃO MUNDIAL DE SAÚDE. CID-10: Classificação Estatística internacional de doenças e problemas relacionados à saúde. São Paulo: Edusp, 2003.

PIEK, J. P.; BAYNAM, G. B.; BARRET, N. C. The relationship between fine and gross motor ability, self-perceptions and selfworth in children and adolescents. Hum. Mov. Sci., v. 25, p.65-76, 2006.

POLATAJKO, H.; J.; CANTIN. N. Developmental coordination disorder (dyspraxia): an overview of the state of the art. Semin. Pediatr. Neurol., v. 12, p. 250-258, 2006.

POULSEN, A. A.; ZIVIANI, J. M.; JOHNSON, H.; CUSKELLY, M. Loneliness and life satisfaction of boys with developmental coordination disorder: the impact of leisure participation and perceived freedom in leisure. Hum. Mov. Sci., v. 27, p. 325-343, 2008 .

PRADO, M.S.; MAGALHÃES, L.C.; WILSON, B.N. Cross cultural translation of the Developmental Coordination Disorder Questionnaire for Brazilian children. Rev. Bras. Fisioter.,v.13, n. 3, p., 2009.

RASMUSSEN, P.; GILLBERG, C. Natural outcome of ADHD with developmental coordination disorder at age 22 years: A controlled, longitudinal, community-based study. J. Am. Acad. Child. Adolesc. Psychiatry, v. 39, n. 11, p. 1424-1431, 2000.

SEGAL, R.; MANDICH, A.; POLATAJKO, H.; COOK, J. V. Stigma and its management: a pilot study of parental perceptions of the experiences of children with developmental coordination disorder. Am. J. Occup. Ther., v. 56, n. 4, p. 422-428, 2002.

SOUZA, C.; FERREIRA, L.; CATUZZO, M.; CORRÊA, U. C. $\mathrm{O}$ teste $\mathrm{ABC}$ do movimento em crianças de ambientes diferentes. Rev. Port. Cien. Desp., v. 7, n. 1, p. 36-47, 2006.

STEPHENSON, E. A.; CHESSON, R. A. "Always the guiding hand": parents' accounts of the long-term implications of developmental co-ordination disorder for their children and families. Child Care Health Develop., v. 34, n. 3, p. 335-343, 2008.

SUGDEN, D. A.; CHAMBERS, M. E. Intervention in children with developmental coordination disorder: The role of parents and teachers. Br. J. Educ. Psychol., v. 73, n. 4, p. 545-561, 2003.

WILSON, B. N.; KAPLAN, B. J.; CRAWFORD, S. G.; CAMPBELL, A., DEWEY, D. Reliability and validity of a parent questionnaire on childhood motor skills. Am. J. Occup. Ther., v. 54, n. 5, p. 484-493, 2000. 